\title{
Evaluation of P-Delta instability in hardening oscillators using dynamical systems approach
}

\author{
Shivang Pathak ${ }^{\mathrm{a}}$, Simon Watt ${ }^{\mathrm{b}}$, Amar Khennane ${ }^{\mathrm{a}}$ and Safat Al-Deen ${ }^{\mathrm{a}}$ \\ ${ }^{a}$ School of Engineering and Information Technology, UNSW Canberra, Canberra ACT 2600, Australia. \\ ${ }^{b}$ School of Science, UNSW Canberra, Canberra ACT 2600, Australia. \\ Email: shivang.pathak@student.adfa.edu.au
}

\begin{abstract}
Construction in earthquake prone areas is an expensive task, as the structural design warrants high material consumption. This is partly due to the fact that the seismic design philosophy is based on the concept of stability through energy dissipation. The energy dissipation is conventionally achieved by controlled plastifi-cation and hysteresis of the structural elements that require high quantities of reinforcements and sophisticated detailing, in both steel and concrete structures. Further, due to inherent uncertainty in the occurrence and the characteristics of earthquakes and also in the current simulation models, a conservative design is essential that can provide a sufficient margin of safety against failure. In the last two decades, however, there is a push towards developing performance designs. This new evolved philosophy of seismic design aims to quantify the uncertainties and the unknown aspects of the design to reduce the margins of safety, while sustaining equally high reliability. As a result, this leads to designs with low requirements of material consumption, thereby making them economic. Consequently, the complexity in the design process increases in almost every aspect, right from quantification of uncertainty by performing Monte-Carlo simulations to developing high-fidelity models that can incorporate all forms of non-linearity in the design. Moreover, to reduce the margins of safety, it becomes imperative to accurately estimate the point of failure or structural collapse capacity. However, currently under the Performance Based Earthquake Engineering (PBEE) framework, the collapse capacity is not evaluated corresponding the the actual dynamic instability in the structural system. Instead, it is estimated corresponding to subjective threshold values of engineering demand parameters, such as lateral deformation. Therefore, in the current paper, a novel-approach is presented that uses dynamical system theory for evaluat-ing dynamic instability in a structure that can be used to accurately estimate its collapse capacity. A P-Delta instability is the dynamic instability that occurs when gravity loads magnify the force demand due to the ge-ometry of the deformed structure, leading to high overturning moments on the base. This is widely studied under mainstream structural analysis. For simplicity, a single-degree-of-freedom (SDOF) system is studied. Therefore, the current work is targeted towards the structures that can be idealised as an SDOF system. The dynamic instability leading to "structural collapse" is defined when the real part of the dominant eigenvalue of the oscillator system becomes positive and remains positive until large deformations occurs. The current study uses harmonic excitations for evaluating dynamic instability and therefore acts as a precursor to a larger study aimed at evaluating mathematical instability in structures under the effects of seismic ground motions.
\end{abstract}

Keywords: P-Delta instability, Dynamical system, Performance based earthquake engineering (PBEE) design, Collapse criterion 


\section{INTRODUCTION}

Performance design for structures under earthquake requires evaluation of their collapse capacities [Building Seismic Safety Council, 2009; FEMA-356, 2000; ASCE, 2014; Zareian et al., 2010; FEMA, 2009; Zareian and Krawinkler, 2007; Krawinkler and Zareian, 2007]. However, the estimate of collapse capacity is sensitive to the method employed for its evaluation and directly impacts the risk of structural collapse |Deniz et al. 2018|. In general, collapse capacity under ground motions is defined as the maximum resistance offered by the structure just before the point when it fails to sustain the originally imposed gravity loads [Ibarra and Krawinkler, 2005]. This can be alternatively interpreted as the maximum resistance offered to avoid dynamic instability. The maximum resistance is typically evaluated in terms of an engineering demand parameter (EDP) such as maximum lateral deformation or lateral drift, also regarded as the damage measure (DM) or in terms of spectral acceleration, $S_{a}$, regarded as the intensity measure (IM). In conventional practice, however, instead of evaluating collapse corresponding to the state of dynamic instability, it is indirectly evaluated by imposing a set of IM/DM rules [Vamvatsikos and Cornell, 2002], such as when the drift changes by more than 10\%. However, this will vary in different structures and thus subjective in nature [Deniz et al., 2017; Bernal, 1992]. Hence, as an improvement, energy methods have emerged that provide a condition for collapse based on dissipated and supplied energies into the structure [Deniz et al., 2017; Zhou and Li, 2017]. Accordingly, the dynamic instability (interpreted as collapse) is defined when the gravitational energy exceeds the supplied earthquake energy [Deniz et al. 2017]. Nevertheless, though the energy methods provide a holistic description of the structure, they rely on occurrence of large deformations, making them act as lagging indicators of collapse. Moreover, they too do not explicitly evaluate the mathematical dynamic instability.

The above problem has been widely studied using single-degree-of-freedom (SDOF) oscillator systems [Villaverde, 2007]. While it uses a lumped mass technique of modelling, SDOF idealisation is only considered suitable for the structures exhibiting a predominant first mode behaviour. Most of the studies base the condition of dynamic instability on the tangent stiffness (force resistance per unit deformation) matrix of the structural system |Araki and Hjelmstad, 2000|. Once the tangent stiffness matrix assumes a negative value or becomes singular, the structure is assumed to become dynamically unstable. However, this condition cannot be considered as sufficient since the SDOF structure may regain stability upon unloading |Bernal, 1992; Araki and Hjelmstad, 2000|. Moreover, the stiffness matrix does not explain the complete dynamics of the system and therefore criteria such as when it becomes singular [Deniz et al., 2017; Villaverde, 2007] or when the eigenvalues become negative [Bažant and Jiŕasek, 1996] do not sufficiently define dynamic instability.

Specifically, for evaluation of instability under seismic ground motion, Araki and Hjelmstad [2000| proposed a set of collapse criteria, when the minimum eigenvalue of the Hessian matrix of the total potential energy of the system becomes negative, while the direction of loading is in the same direction as forcing. However, instability may occur when the loading and forcing are not in the same direction. Further, it is assumed that unloading only occurs under free oscillations of the structure, which may not be true. With these limitations, this set of collapse criteria also cannot predict dynamic instability under varied ground motion excitations.

A dynamical systems approach was made by Challamel and Gilles [2007], where they investigated an elasticperfectly-plastic SDOF system under harmonic excitations. To understand the dynamics and establish domains of instability, they segregated the oscillator's response in elastic and plastic regimes. Nonetheless, although their investigation was quite comprehensive, segregation of the response into two unique regimes was not possible for hysteretic hardening oscillators.

It can be concluded that a criterion to mathematically define P-Delta instability it is essential to accurately evaluate collapse capacity of structures that undergo hysteresis. In the current study, dynamical systems theory based approach is undertaken to define instantaneous instability in SDOF oscillator systems (similar to [Bažant and Jiŕasek, 1996]). Subsequently, using the information of instantaneous instability, a new criterion is proposed that defines global instability. For illustration, a bilinear hardening SDOF system is considered under harmonic base excitation. For such non-deteriorating system, the stiffness repeatedly alternates and thus it forms a case of non-autonomous non-smooth system. Owing to this non-linearity, the governing equation of the system changes as the stiffness varies. Therefore, for assessing stability, individual governing differential equations are considered as an ensemble of unique piece-wise systems.

\section{MATHEMATICAL FORMULATION}

The system under consideration is a rigid column of height $25.4 \mathrm{~cm}$ (10 inches) with a $36.3 \mathrm{~kg}$ ( 80 pound) lumped mass on the top, which is attached to a spring, which is attached to a shake table (see Figure 1ı). 
This is an idealisation of the experimental structure tested by Kanvinde [2003]. To emulate the punched hole section at the base of the oscillator, the base node is assumed to behave as a zero-length rotational spring that follows a bilinear hysteretic material model, shown in Figure 1 j. It describes the resisting moment that the spring offers against rotation when the oscillator loads/unloads. The modelling parameters of the hysteretic model are provided in Table 1 . The other end of the rotational spring is attached to the base and is restrained in all degrees of freedom. The oscillator is excited under base acceleration and the governing equation of motion is given as:

$$
I \ddot{\theta}+\gamma h \dot{\theta}+\tau(\theta)=-\left(m \ddot{u}_{g}\right) \cdot h \cos \theta .
$$

where $h$ is the height of the column, $m$ is the mass on the top of the column, $I=m h^{2}$ (mass moment of inertia), $\gamma=c \times h$ (damping coefficient * height), $\tau(\theta)=k \theta-P h \sin \theta$ (net restoring moment), $P=m g$ is the weight on the top of the oscillator, $g$ is the acceleration due to gravity; $k=k(\theta)$ rotational stiffness of the spring; $\theta=\theta(t)$ is the time dependent rotation of the structure, $\dot{\theta}=\dot{\theta}(t)$ is the angular velocity, $\ddot{\theta}=\ddot{\theta}(t)$ is the angular acceleration and $\ddot{u}_{g}$ is the base acceleration.

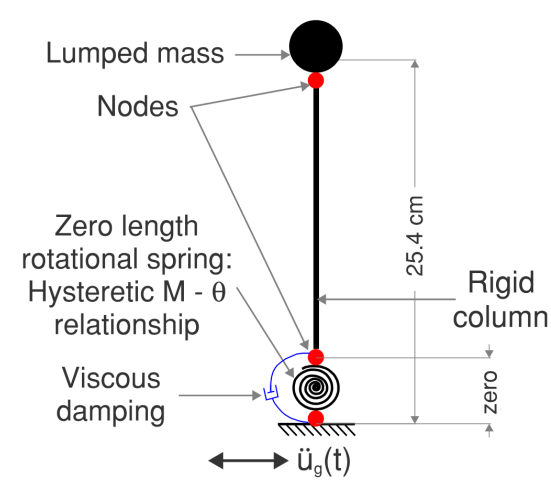

(a)

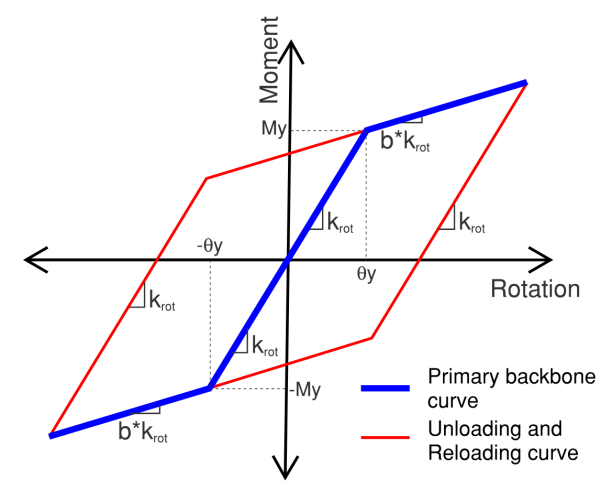

(b)

Figure 1. Single-degree-of-freedom oscillator (a) Mathematical model; and (b) Hysteretic model of the zero length spring at its base.

Segregating the angular acceleration, the governing equation of the oscillator can be written as:

$$
\ddot{\theta}=-\frac{\ddot{u}_{g}}{h} \cos \theta-\frac{\gamma h}{I} \dot{\theta}-\frac{1}{I}(k \theta-P h \sin \theta),
$$

and rewritten as a first order system:

$$
\begin{aligned}
& \dot{y_{1}}=y_{2}, \\
& \dot{y_{2}}=-\frac{\ddot{u}_{g}}{h} \cos y_{1}-\frac{\gamma h}{I} y_{2}-\frac{1}{I}\left(k y_{1}-P h \sin y_{1}\right),
\end{aligned}
$$

with $y_{1} \equiv \theta$. The assumption made is that that ground motion is piecewise constant over each time period between observations. The equilibrium points $\left(y_{1}^{e q}\right.$ and $\left.y_{2}^{e q}\right)$ for this system at any time $t$ are found when $\dot{y}_{1}(t)=0$ and $\dot{y_{2}}(t)=0$, respectively. This yields $y_{2}^{e q}=0$ and $y_{1}^{e q}$ satisfies the equation:

$$
-\frac{\ddot{u}_{g}}{h} \cos y_{1}^{e q}-\frac{1}{I}\left(k y_{1}^{e q}-P h \sin y_{1}^{e q}\right)=0
$$

With a time-dependent ground motion, as given by $\ddot{u}_{g}$, the equation (1) is a non-autonomous model. As discussed by Josić and Rosenbaum [2008], the eigenvalues of the Jacobian of the non-autonomous system do not necessarily determine the stability of the system. However, we postulate that the behaviour of this particular system in the neighbourhood of the equilibrium point can be determined by the eigenvalues of the Jacobian of the system evaluated at the equilibrium point, where the Jacobian $\mathbf{J}$ is:

$$
\mathbf{J}=\left[\begin{array}{cc}
0 & 1 \\
\frac{\ddot{u}_{g}}{h} \sin y_{1}^{e q}-\frac{1}{I}\left(k-P h \cos y_{1}^{e q}\right) & -\frac{\gamma h}{I}
\end{array}\right]
$$


The eigenvalues of the system at the equilibrium point are denoted $\lambda_{1}, \lambda_{2}$. Therefore, by evaluating the nature of the equilibrium point governed by eigenvalues, the instantaneous stability of the oscillator can be determined. However, since the governing equation of the system changes (due varying rotational stiffness $k$ ), the nature of the equilibrium point at instant $t_{i-1}$ does not guarantee stability at instant $t_{i}$. This necessitates continuous assessment of the nature of the equilibrium points at every time step to determine "global" instability. A similar approach has been taken by Bažant and Jiŕasek |1996| where they considered seismic damage in frames.

Table 1. Modelling parameters for the hysteretic material model [Kanvinde, 2003].

\begin{tabular}{|l|c|}
\hline Hysteretic Model in OpenSees & Steel-01 (Bi-linear hysteretic) \\
\hline Yield Moment $(\mathbf{M y})$ & $27.1 \mathrm{~N}-\mathrm{m}$ \\
\hline Initial rotational stiffness $\left(k_{r o t}\right)$ & $903.87 \mathrm{~N}-\mathrm{m} / \mathrm{rad}$. \\
\hline Hardening coefficient $(\mathbf{b})$ & $2 \%$ of $k_{r o t}$ \\
\hline Unloading stiffness $\left(k_{u n}=k_{r o t}\right)$ & $903.87 \mathrm{~N}-\mathrm{m} / \mathrm{rad}$. \\
\hline
\end{tabular}

\subsection{Nature of equilibrium points}

The nature of the equilibrium point is evaluated based on the system eigenvalues $\lambda_{1}, \lambda_{2}$ are given as:

$$
\lambda_{1}, \lambda_{2}=\frac{J_{22}}{2} \pm \sqrt{\left(\frac{J_{22}}{2}\right)^{2}+J_{21}} .
$$

where $J_{21}$ and $J_{22}$ are elements of the Jacobian matrix $\mathbf{J}$ given by equation (6). The set $\mathcal{S}$ representing all the equilibrium points is given as:

$$
\mathcal{S}=\bigcup_{i=1}^{i=n_{e q}}\left\{\left.y_{1}^{e q}\right|_{i},\left.y_{2}^{e q}\right|_{i}\right\}=\bigcup_{i=1}^{i=n_{e q}}\left(E_{i}\right),
$$

where $n^{e q}$ is the total number of equilibrium points observed when the system is analysed.

As the oscillator is a simplified idealisation of a conventional structural system, strictly positive damping is considered, making $J_{22}<0$. Thus, there can be only two possible types of behaviour. If $\left[\left(J_{22} / 2\right)^{2}+J_{21}\right]<0$, then the equilibrium point is a stable sink. Whereas, if $\left[\left(J_{22} / 2\right)^{2}+J_{21}\right]>0$, then the equilibrium point is an unstable saddle node. We denote points of instantaneous instability of the system occurs where the equilibrium point changes from a stable sink at $t=t_{i}$ to an unstable saddle node at $t=t_{i+1}$.

\subsection{Criterion for structural collapse}

To determine the state of dynamic instability of the system that results in structural collapse, the first point when the saddle node instability appears is selected. However, it is possible that the oscillator regains its stability and the saddle node instability turns back into a stable spiral sink behaviour. This previously selected first point is then discarded. If the oscillator fails to regain its stability and sustains the instantaneous saddle node instability state while undergoing high deformations, this selected first point is then considered as the point of "global" instability or collapse. This will define the collapse criterion based on the dynamic stability approach.

This method of continuous assessment of the instantaneous stability of the system can be utilised to determine its global instability in context of structural collapse. It should be noted that it is possible that even after the point of collapse, the oscillator may again show stable behaviour (spiral sinks). This is because the governing differential equation may provide a stable solution mathematically, but physically the oscillator has already collapsed.

\section{IDENTIFYING INSTABILITY IN THE OSCILLATOR}

The dynamics of the described oscillator system is studied under a sinusoidal acceleration time history. Based on the material parameters shown in Table 1, the modal analysis results in the fundamental circular frequency, 
$\omega_{f}=\sqrt{k_{\text {rot }} /\left(m h^{2}\right)}=19.6571 \mathrm{rad} / \mathrm{sec}$. The forcing sinusoidal time history has an amplitude of 0.3 times of ground acceleration and a circular frequency $\left(\omega_{\text {ext }}\right)$, one-fifth of the fundamental frequency of the oscillator system $\left(\omega_{\text {ext }}=\omega_{f} / 5\right)$.

The time history analysis is carried out using the OpenSees (Open System for earthquake engineering simulation) program [McKenna et al. 2007; Mazzoni et al., 2006]. A mass proportional Rayleigh damping of $2 \%$ is assumed and co-rotation formulation is used to account for the non-linear geometric effects. To illustrate the difference in the non-collapse case and collapse case, two types of amplitude scaling is performed for the input sinusoidal time history. Under amplitude scaling by a factor of $S F=0.85$, the response of the system is shown in Figure 2. The horizontal deformation of the oscillator is constrained (Figure $2 a$ ) and so is the base angle (Figure 23 ), while the vertical deformation is almost negligible. The dominant eigenvalue of the system, though fluctuates from negative to positive (Figure 2a) indicating instantaneous instability, it finally settles to a negative value as the system approaches a steady-state oscillation, indicating stability. On the other hand, under no scaling $(S F=1.00)$, both the horizontal and vertical deformation (Figure $3 \imath$ ) and base angle (Figure 3 ) rise significantly as the oscillator "collapses". From Figure 3:, the dominant eigenvalue first becomes positive after 0.2 seconds and the system loses instantaneous stability. However, the system regains its stability at around 0.9 seconds. It can be seen from Figure 3 a that the deformations remain constrained during this period. However, the system again enters the unstable state at around 1.5 seconds and experiences high deformations and finally completely collapses at 2.1 seconds as the base angle becomes greater than $\pi / 2$ radians.

(a) Top node deformations

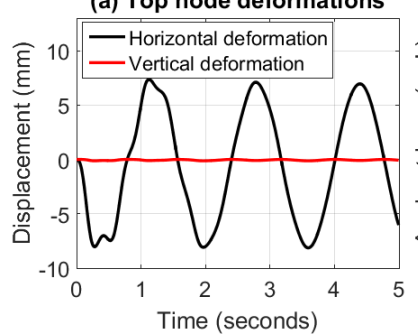

(d) 4-Dimensional decription of instantaneous stability $(0.255 \mathrm{~g}$ amplitude

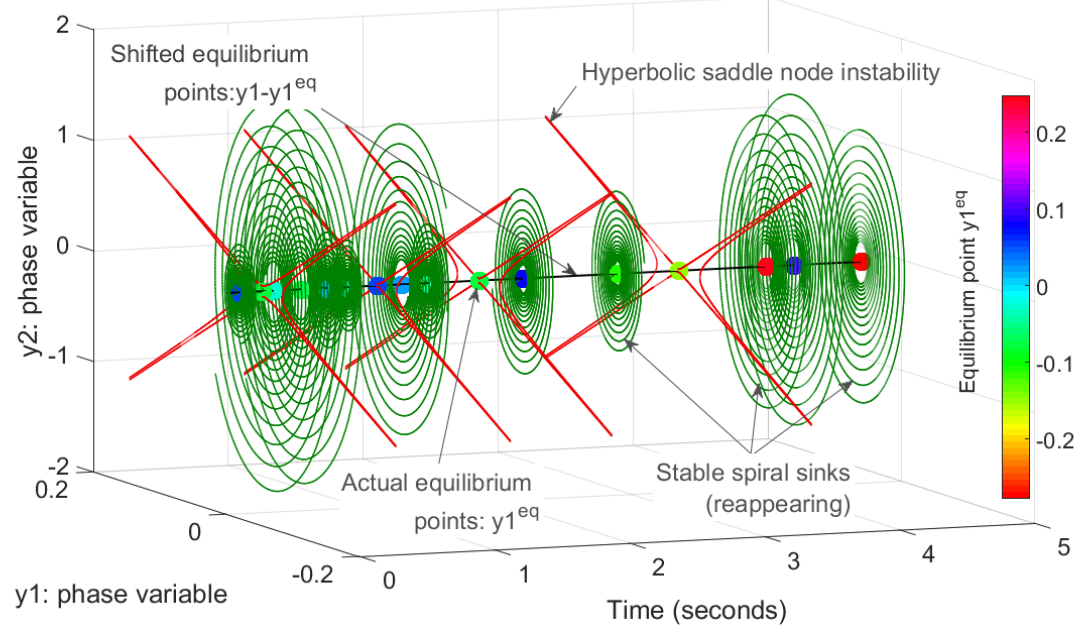

Figure 2. Response of the oscillator system under scaled harmonic excitation $(\mathrm{SF}=0.85)$.

As discussed previously, the first point when the equilibrium point loses instantaneous stability before the system experiences high deformation is selected as the point of "global" instability or onset of collapse. The triangle marker on the deformation time history (Figure 3a) represents the collapse capacity in term of response. Hence, the predicted drift at collapse for the oscillator is roughly $30 \%$, whereas the vertical deformation is only $12 \mathrm{~mm}$. It can be observed that since the dynamic instability is predicted mathematically, the exact onset of collapse can be determined. Therefore, unlike energy methods that rely on gravitational energy (which in 

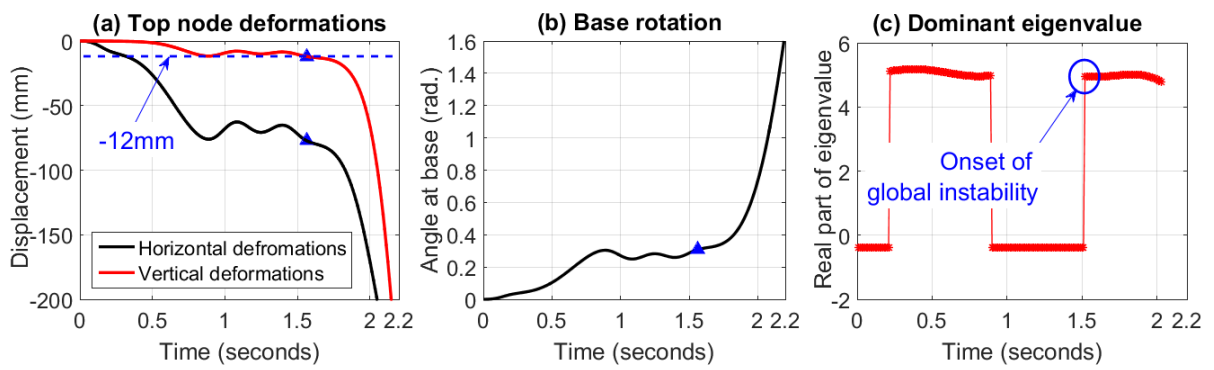

(d) 4-Dimensional decription of instantaneous stability $(0.3 \mathrm{~g}$ amplitude)

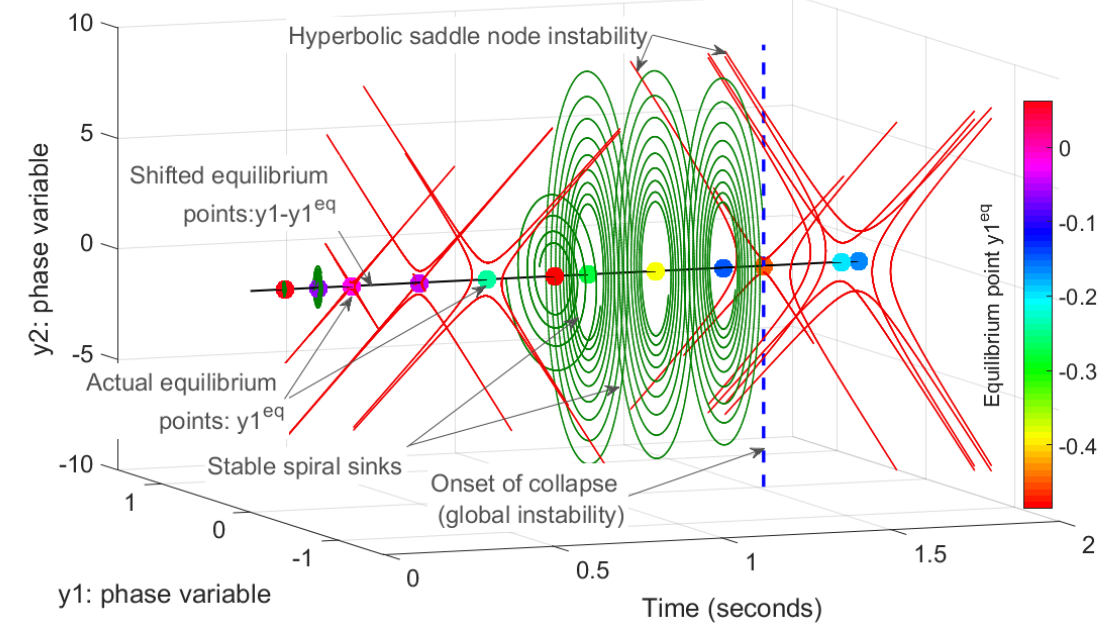

Figure 3. Response of the oscillator system under harmonic excitation $(\mathrm{SF}=1.00)$. The blue triangles denote the onset of global instability.

turn depends upon vertical deformations), this approach predicts collapse without undergoing any significant vertical deformations. Thus, the dynamic stability based criterion serves as a "leading" collapse indicator.

Further, the evolution of the stability states can be visualised by 4-dimensional phase portrait plots, where the three spatial dimensions are time, the angle and the angular velocity. The fourth dimension is the colour of the circles on the centre line, representing equilibrium angle, $y_{1}^{e q}$. Since the dynamics of the system is studied as an ensemble of different sub-systems, each sub-system at every time step can be regarded as an autonomous system. The instantaneous governing differential equation is based on the stiffness and the external force at this particular time step. The equilibrium points can be found at every such step and the instantaneous stability in the neighbourhood of the equilibrium position can be visualised as shown in Figure $2 d$ and Figure $3 d$ for the two cases under consideration. The evolution of phase variables at every time step, for each sub-system, about the equilibrium point can be seen in the three spatial dimensions and the nature of the equilibrium point is shown in the fourth "colour" dimension.

Shown in Figure $2 \mathrm{~d}$, for the response under scaled time history $(\mathrm{SF}=0.85)$, the system shows momentary unstable behaviour, shown by hyperbolic saddles. However, since the oscillator stays stable for the most part, spiral sinks appear predominantly. On the other hand, Figure 3.1 shows the system undergoing instantaneous stability changes at $0.25,0.9$ and 1.5 seconds. After this point, the system does not regain stability and hyperbolic saddles can be seen beyond 1.5 seconds indicating unstable behaviour and collapse.

\section{Conclusions}

This paper presents a dynamical systems approach for evaluating instability in a single degree of freedom oscillator under harmonic excitations. The global instability that induces collapse in the structure is studied by continuously evaluating the stability state of the instantaneous equilibrium points. A four dimensional representation of the phase portrait developed to visualise the evolution of the stability in the neighbourhood of the equilibrium state. The collapse is detected when the behaviour changes from a sink to a saddle node and remains in this state as the oscillator undergoes high deformations. Thus, using the proposed mathematical 
interpretation of the instability, collapse capacity of the structure can be accurately determined. In this study, only simple illustrations are presented to show a proof of concept, that are first few steps towards a wider goal of mathematically evaluating instability in complex structures under seismic ground motions. It should also be noted that the main caveat is the system under consideration is a non-autonomous system and therefore the stability of the system is not necessarily determined by the stability of the linear approximation to the system. We evaluate the effectiveness of this method a posteriori.

\section{ACKNOWledgements}

The authors are grateful for the financial support provided by the University of New South Wales.

\section{REFERENCES}

Araki, Y. and K. Hjelmstad (2000). Criteria for assessing dynamic collapse of elastoplastic structural systems. Earthquake engineering \& structural dynamics 29, 1177-1198.

ASCE (2014). Seismic Evaluation and Retrofit of Existing Buildings. ASCE/SEI Standard 41-14.

Bažant, Z. and M. Jiŕasek (1996). Softening-induced dynamic localization instability : Seismic damage in frames. Journal of Engineering Mechanics ASCE 122(12), 1149-1152.

Bernal, D. (1992). Instability of buildings subjected to earthquakes. Journal of Structural Engineering 118, 2239-2260.

Building Seismic Safety Council (2009). NEHRP recommended seismic provisions for new buildings and other structures (FEMA P-750). Washington, DC: Federal Emergency Management Agency.

Challamel, N. and G. Gilles (2007). Stability and dynamics of a harmonically excited elastic-perfectly plastic oscillator. Journal of Sound and Vibration 301, 608-634.

Deniz, D., J. Song, and J. F. Hajjar (2017). Energy-based seismic collapse criterion for ductile planar structural frames. Engineering Structures 141, 1-13.

Deniz, D., J. Song, and J. F. Hajjar (2018). Energy-based sidesway collapse fragilities for ductile structural frames under earthquake loadings. Engineering Structures 174, 282-294.

FEMA (2009). Quantification of seismic performance factors, prepared by the applied technology council (ATC), Volume Report No. FEMA-P695. Washington, DC.

FEMA-356 (2000). NEHRP Guidelines for the Seismic Rehabilitation of Buildings. Building Seismic Safety Council Washington, DC.

Ibarra, L. F. and H. Krawinkler (2005). Global collapse of frame structures under seismic excitations. Pacific Earthquake Engineering Research Center Berkeley, CA.

Josić, K. and R. Rosenbaum (2008). Unstable solutions of nonautonomous linear differential equations. SIAM Review 50, 570-584.

Kanvinde, A. M. (2003). Methods to evaluate the dynamic stability of structures-shake table tests and nonlinear dynamic analyses. In EERI Paper Competition 2003 Winner. Proceedings of the EERI Meeting, Portland.

Krawinkler, H. and F. Zareian (2007). Prediction of collapsehow realistic and practical is it, and what can we learn from it? The Structural Design of Tall and Special Buildings 16, 633-653.

Mazzoni, S., F. McKenna, M. H. Scott, and G. L. Fenves (2006). OpenSees command language manual, Pacific Earthquake Engineering Research Center, Berkeley.

McKenna, F., G. Fenves, and M. Scott (2007). Open system for earthquake engineering simulation. Pacific Earthquake Engineering Research Center, Berkeley.

Vamvatsikos, D. and C. A. Cornell (2002). Incremental dynamic analysis. Earthquake Engineering and Structural Dynamics 31, 491-514.

Villaverde, R. (2007). Methods to assess the seismic collapse capacity of building structures: state of the art. Journal of Structural Engineering 133, 57-66.

Zareian, F. and H. Krawinkler (2007). Assessment of probability of collapse and design for collapse safety. Earthquake Engineering \& Structural Dynamics 36, 1901-1914.

Zareian, F., H. Krawinkler, L. Ibarra, and D. Lignos (2010). Basic concepts and performance measures in prediction of collapse of buildings under earthquake ground motions. The Structural Design of Tall and Special Buildings 19, 167-181.

Zhou, H. and J. Li (2017). Effective energy criterion for collapse of deteriorating structural systems. Journal of Engineering Mechanics 143, p. 04017135. 